\title{
Theory for Magnetic-Field-Driven 3D Metal-Insulator Transitions in the Quantum Limit
}

\author{
Peng-Lu Zhao, ${ }^{1}$ Hai-Zhou Lu, ${ }^{1,2, *}$ and X. C. Xie ${ }^{3,4,5}$ \\ ${ }^{1}$ Shenzhen Institute for Quantum Science and Engineering and Department of Physics, \\ Southern University of Science and Technology (SUSTech), Shenzhen 518055, China \\ ${ }^{2}$ Shenzhen Key Laboratory of Quantum Science and Engineering, Shenzhen 518055, China \\ ${ }^{3}$ International Center for Quantum Materials, School of Physics, Peking University, Beijing 100871, China \\ ${ }^{4}$ CAS Center for Excellence in Topological Quantum Computation, \\ University of Chinese Academy of Sciences, Beijing 100190, China \\ ${ }^{5}$ Beijing Academy of Quantum Information Sciences, West Building 3, \\ No. 10, Xibeiwang East Road, Haidian District, Beijing 100193, China
}

(Dated: July 29, 2021)

\begin{abstract}
Metal-insulator transitions driven by magnetic fields have been extensively studied in 2D, but a $3 \mathrm{D}$ theory is still lacking. Motivated by recent experiments, we develop a scaling theory for the metal-insulator transitions in the strong-magnetic-field quantum limit of a 3D system. By using a renormalization-group calculation to treat electron-electron interactions, electron-phonon interactions, and disorder on the same footing, we obtain the critical exponent that characterizes the scaling relations of the resistivity to temperature and magnetic field. By comparing the critical exponent with those in a recent experiment [F. Tang et al., Nature (London) 569, 537 (2019)], we conclude that the insulating ground state was not only a charge-density wave driven by electronphonon interactions but also coexisting with strong electron-electron interactions and backscattering disorder. We also propose a current-scaling experiment for further verification. Our theory will be helpful for exploring the emergent territory of 3D metal-insulator transitions under strong magnetic fields.
\end{abstract}

Introduction.-Metal-insulator transition is a fascinating problem in condensed matter physics because of its rich mechanisms [1-3]. There have been extensive studies on 2D metal-insulator transitions in magnetic fields [1-17], but a 3D theory is still lacking, despite that recent experiments show that magnetic fields can drive metal-insulator transitions in 3D systems [18-22] with characteristics of quantum phase transitions [23-25] [see Fig. 1(a)], in particular, in the strong-field quantum limit of a topological insulator [21, 22]. It is challenging to determine the insulating ground states in strong magnetic fields, because magnetic fields reduce the effective dimension, leading to stronger interactions and related instabilities, such as charge or spin density wave, Wigner crystal, Anderson localization, etc. [25-32].

In this Letter, we develop a scaling theory for the metal-insulator transition in the quantum limit of a 3D topological insulator under strong magnetic fields (see Fig. 2). Our theory agrees well with recent experiments [21]. The quantum phase transition is governed by universal relations described by critical exponents [23] [Fig. 1(a)], corresponding to various instabilities and universal classes $[25,29,33-38]$. With the help of a renormalization-group calculation, we find the dynamical critical exponents $z$ and correlation length exponents $\nu$ for the candidate instabilities. Based on them, we build the scaling relations of the resistivity to temperature and magnetic field, described by a critical exponent $\xi$. We find $\xi$ for five cases (Table I). By comparing with the experimentally measured $\xi=5.5[21]$, we conclude that the insulating ground state is a charge-density wave not only (a) $\uparrow T$
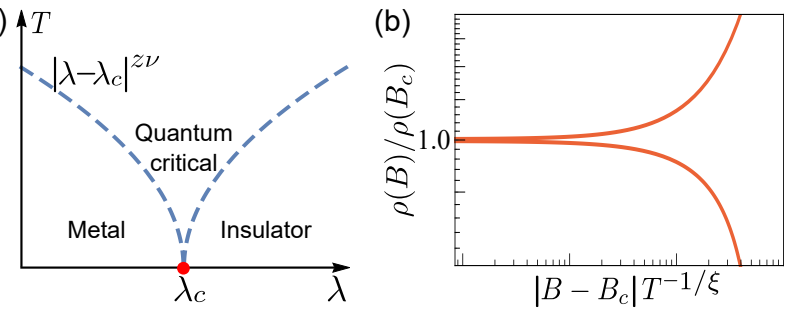

FIG. 1. (a) A quantum phase transition can be described by a nonthermal parameter $\lambda$ that converges to $\lambda_{c}$ at the quantum critical point. The boundaries (dashed curves) of the quantum critical region are described by $T \propto\left|\lambda-\lambda_{c}\right|^{z \nu}$, where $z$ is the dynamical critical exponent and $\nu$ is the correlation length exponent. (b) The magnetic field $B$ can serve as $\lambda$, and $z$ and $\nu$ can be used to construct a measurable critical exponent $\xi$ that describes the resistivity as a scaling function of the magnetic field and temperature, e.g., $\rho(B) / \rho\left(B_{c}\right)=f\left(\left|B-B_{c}\right| / T^{1 / \xi}\right)$, where $f(x)$ is a scaling function with $f(0)=1$.

driven by electron-phonon interactions but also requiring strong electron-electron interactions and backscattering disorder. We also propose a current-scaling experiment for further verification, by fitting the dynamical critical exponent and correlation length exponent. Our theory will be helpful for the emergent territory of 3D metalinsulator transitions under strong magnetic fields.

Model for $3 D$ systems in strong fields. $-3 \mathrm{D}$ insulators and metals can be generically described by a Dirac model [41]

$$
\mathcal{H}=m(\mathbf{k}) \tau_{z} \sigma_{0}+v_{x} k_{x} \tau_{x} \sigma_{z}+v_{y} k_{y} \tau_{y} \sigma_{0}+v_{z} k_{z} \tau_{x} \sigma_{x},(1)
$$


TABLE I. Comparison between experiments [21] and our theory on the critical exponent $\xi$ that describes the scaling relations of the resistivity to temperature and magnetic field, for different dominant and coexisting interactions and disorder. Theoretically, $\xi$ is a product of the dynamical critical exponents $z$ and correlation length exponents $\nu$. In the experiments, the metal-insulator transition happens at a critical magnetic field of $B_{c}=6.71 \mathrm{~T}$, where an incommensurate charge-density wave dominates the ground state according to our theory [39], different from the previous work [40] for $B \in[1.7,2.1] \mathrm{T}$, where the Hall resistivity plateau indicates a commensurate charge-density wave as the ground state.

\begin{tabular}{|c|c|c|c|c|c|c|c|}
\hline $\begin{array}{l}\text { Insulating } \\
\text { phases }\end{array}$ & & Dominant & \multicolumn{2}{|c|}{ Coexisting } & $z$ & $\nu$ & $\xi$ \\
\hline $\begin{array}{l}\text { Charge- } \\
\text { density } \\
\text { wave }\end{array}$ & & $\begin{array}{l}\text { Electron- } \\
\text { phonon }\end{array}$ & \multicolumn{2}{|c|}{$\begin{array}{l}\text { Electron- } \\
\text { electron }\end{array}$} & 1 & 1.5 & 1.5 \\
\hline $\begin{array}{l}\text { Anderson } \\
\text { insulator }\end{array}$ & & $\begin{array}{l}\text { Forward- } \\
\text { scattering } \\
\text { disorder }\end{array}$ & \multicolumn{2}{|c|}{$\begin{array}{l}\text { Electron-phonon } \\
\text { Electron- } \\
\text { electron }\end{array}$} & 1 & 2 & 2 \\
\hline $\begin{array}{l}\text { Charge- } \\
\text { density } \\
\text { wave }\end{array}$ & & $\begin{array}{l}\text { Electron } \\
\text {-phonon }\end{array}$ & \multicolumn{2}{|c|}{$\begin{array}{l}\text { Backscattering } \\
\text { disorder } \\
\text { Weak electron } \\
\text {-electron }\end{array}$} & 1.5 & 3 & 4.5 \\
\hline $\begin{array}{l}\text { Wigner } \\
\text { crystal }\end{array}$ & & $\begin{array}{l}\text { Electron } \\
\text {-electron }\end{array}$ & \multicolumn{2}{|c|}{$\begin{array}{l}\text { Backscattering } \\
\text { disorder } \\
\text { Electron- } \\
\text { electron }\end{array}$} & 2 & 1 & 2 \\
\hline $\begin{array}{l}\text { Charge- } \\
\text { density } \\
\text { wave }\end{array}$ & & $\begin{array}{l}\text { Electron } \\
\text {-phonon }\end{array}$ & \multicolumn{2}{|c|}{$\begin{array}{l}\text { Backscattering } \\
\text { disorder } \\
\text { Strong electron } \\
\text {-electron }\end{array}$} & 2 & 3 & 6 \\
\hline \multicolumn{8}{|c|}{ Experiment } \\
\hline Sample & 1 & 2 & 4 & $\begin{array}{l}\text { Mean value } \\
\text { (all samples) }\end{array}$ & & $\begin{array}{l}\text { Tean v } \\
(2,3,2\end{array}$ & \\
\hline$\xi$ & 3.95 & $5 \quad 6.06 \quad 5.78$ & 6.25 & $5.5 \pm 1.1$ & & $6.0 \pm$ & \\
\hline
\end{tabular}

where $\mathbf{k}=\left(k_{x}, k_{y}, k_{z}\right)$ is the wave vector, $\tau$ and $\sigma$ are Pauli matrices for pseudo and real spin, respectively, $v_{x, y, z}$ are parameters for the "Fermi velocities," and $m(\mathbf{k})$ stands for the "Dirac mass" $[42,43]$. We will focus on the quantum limit in strong fields, where a $z$ direction magnetic field splits the energy spectrum into a series of $1 \mathrm{D}$ bands of Landau levels dispersing with $k_{z}$ and only the lowest Landau band $0+$ is occupied (Fig. 2). An effective free Hamiltonian for the lowest Landau band can be found by linearizing the dispersion near the two Fermi wave vectors $\pm k_{F} \mathbf{e}_{z}$ [Fig. 2(b)] as $H_{0}=\sum_{\mathbf{k}} \psi^{\dagger}(\mathbf{k}) v_{F} k_{z} \sigma_{z} \psi(\mathbf{k})$ [39], where $\psi(\mathbf{k})=$ $\left[\psi_{-}(\mathbf{k}), \psi_{+}(\mathbf{k})\right]^{\mathrm{T}}, \psi_{ \pm}^{\dagger}(\mathbf{k})$ and $\psi_{ \pm}(\mathbf{k})$ are the creation and annihilation operators, respectively, near $\mp k_{F}$, and $\mathbf{k}$ is measured from $\pm k_{F} \mathbf{e}_{z}$.

Interactions and disorder.-Electron-electron or electron-phonon interactions can open a charge-densitywave gap near $\pm k_{F}$ [see Fig. 2(c)]. The charge-density (a) no Magnetic field

(b) In magnetic field

(c) With interactions

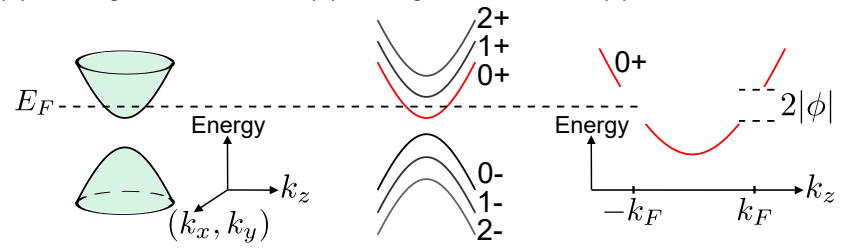

FIG. 2. A $z$-direction magnetic field splits the energy spectrum of a 3D insulator or metal (a) into 1D bands of Landau levels dispersing with the wave vector $k_{z}(\mathrm{~b})$. The $n \pm$ stand for the $n$th Landau bands. In the quantum limit, the Fermi energy $E_{F}$ crosses the lowest Landau band $0+$ at $\pm k_{F}$, which is a metal phase and the starting point of this work. (c) Correlations between electrons near $-k_{F}$ and $k_{F}$ open a gap of size $2|\phi|$ and induce an insulating phase.

wave may induce the Wigner crystal [26, 27]. Also, disorder could induce the Anderson localization [28-30]. We study them on the same footing by using the effective action

$$
\begin{aligned}
S & =\int d^{3} x d \tau\left(\mathcal{L}_{e}+\mathcal{L}_{p}+\mathcal{L}_{b}\right)+\int d^{3} x d \tau d \tau^{\prime} \mathcal{L}_{d}, \\
\mathcal{L}_{e} & =u \psi_{+}^{\dagger} \psi_{+} \psi_{-}^{\dagger} \psi_{-}, \quad \mathcal{L}_{p}=g\left(\phi \psi_{+}^{\dagger} \psi_{-}+\phi^{*} \psi_{-}^{\dagger} \psi_{+}\right), \\
\mathcal{L}_{b} & =\left|\partial_{\tau} \phi\right|^{2}+v_{b}^{2}|\boldsymbol{\partial} \phi|^{2}+r|\phi|^{2} \\
\mathcal{L}_{d} & =-\sum_{i=f, b}\left(\Delta_{i} / 2\right)\left(\psi^{\dagger} \Gamma_{i} \psi\right)_{\tau}\left(\psi^{\dagger} \Gamma_{i} \psi\right)_{\tau^{\prime}} .
\end{aligned}
$$

The Lagrangian $\mathcal{L}_{e}$ describes electron-electron interactions that induce the $2 k_{F}$ instability [44]. $\mathcal{L}_{p}$ represents the coupling between charge-density-wave order parameter $\phi$ and electrons $[32,40,45]$. The order parameter is defined as $\phi=\left(\alpha_{2 k_{F}} / V\right)\left(\left\langle b_{-2 k_{F} \mathbf{e}_{z}}^{\dagger}\right\rangle+\left\langle b_{2 k_{F} \mathbf{e}_{z}}\right\rangle\right)$, where $b_{\mathbf{q}}^{\dagger}$ and $b_{\mathbf{q}}$ are the creation and annihilation operators, respectively, for the phonons with momentum $\mathbf{q}$ and $\alpha_{q}$ measures the electron-phonon coupling strength $[40,45] . \mathcal{L}_{b}$ describes the order parameter dynamics [24] with $r=0$ at the quantum critical point. $\mathcal{L}_{d}$ is the Lagrangian of disorder after being ensemble averaged by means of the replica method [46-48]. The Hamiltonian of disorder takes the form $\mathcal{H}_{\text {dis }}=U_{i}(x) \psi^{\dagger} \Gamma_{i} \psi$, where $U_{i}(x)$ is the impurity potential of a Gaussian white-noise distribution as $\left\langle U_{i}\right\rangle=0$ and $\left\langle U_{i}(\mathbf{x}) U_{j}\left(\mathbf{x}^{\prime}\right)\right\rangle=\Delta_{i} \delta_{i j} \delta\left(\mathbf{x}-\mathbf{x}^{\prime}\right)$. For the forward-scattering disorder, $\Gamma_{i}=\sigma_{0}$, which couples electrons near $k_{F}$ only to those near $k_{F}$ or $-k_{F}$ only to those near $-k_{F}[31,49]$, which may induce the Anderson localization [28-30]. For the backscattering disorder, $\Gamma_{i}=\sigma_{x}+\sigma_{y}$, which couples electrons near $k_{F}$ to those near $-k_{F}$ or $-k_{F}$ to those near $k_{F}[31,49]$. We study these two types of disorder separately.

Renormalization-group equations.-The renormalization group [50-53] is an approach to determine the instabilities and corresponding critical exponents. We perform a Wilsonian momentum-shell renormalizationgroup analysis [54-58] for the model described by Eq. (2). 
The momentum shell is defined as $e^{-\ell} \Lambda<\left|\mathbf{k}_{z}\right|<$ $\Lambda$, where $\ell$ is the running scale parameter. The renormalization-group flow equations are found as [39]

$$
\begin{aligned}
d v_{F} / d \ell & =\left[z-1+2 \beta \gamma^{3} /(\gamma+1)^{2}-a_{1 i} \Delta_{i}\right] v_{F}, \\
d \Delta_{f} / d \ell & =2 \Delta_{f}^{2}+\Delta_{f}-4 \beta \gamma^{3} \Delta_{f} /(\gamma+1)^{2}, \\
d \Delta_{b} / d \ell & =-4 \Delta_{b}^{2}+(1+2 u) \Delta_{b}-\frac{2 \beta \gamma^{2}(2 \gamma+1) \Delta_{b}}{(\gamma+1)^{2}}, \\
d \beta / d \ell & =-2 \beta^{2} \gamma^{2} /(\gamma+1)^{2}+\left(2+2 u-2 a_{2 i} \Delta_{i}\right) \beta, \\
d u / d \ell & =2 u^{2}-2 \beta \gamma^{2} u /(\gamma+1)-a_{3 i} \Delta_{i} u,
\end{aligned}
$$

where $a_{m i}$ is a $3 \times 2$ constant matrix with elements $a_{m i}=(m, 2)$, and $i=f, b$ representing the forward- and backward-scattering disorder, respectively. $z$ is the dynamic exponent, which can be found by fixing $v_{F}$. We have redefined the dimensionless coupling constants as

$$
\begin{aligned}
\text { electron-phonon: } & g^{2} / 4 \pi^{2} \ell_{B}^{2} v_{b}^{3} \Lambda^{2} \rightarrow \beta, \\
\text { electron-electron: } & u / 4 \pi^{2} v_{F} \ell_{B}^{2} \rightarrow u, \\
\text { disorder: } & \Delta_{i} / 2 \pi^{2} v_{F}^{2} \ell_{B}^{2} \Lambda \rightarrow \Delta_{i},
\end{aligned}
$$

where $\ell_{B}=\sqrt{\hbar /(e B)}$ is the magnetic length. Different from nonmagnetic field theories, the integrals in the plain give the Landau degeneracy of the magnetic field [39]. The renormalization-group equations highly depend on the parameter $\gamma \equiv\left|v_{b} / v_{F}\right|$, the ratio of the phonon speed to the Fermi velocity, which does not flow with $\ell$, and $\gamma \approx 1.7 \times 10^{-3}$ from the model parameters [39, 59-61]. Later, our conclusions hold for a range of small $\gamma$.

Charge-density wave without disorder?-For a system with only electron-electron and electron-phonon interactions, there are two fixed points [Fig. 3(a)] at (i) $\left(u_{*}, \beta_{*}\right)=(0,0)$ and $(\mathrm{ii})_{c}\left[0,(1+1 / \gamma)^{2}\right]$. If the phononFermi velocity ratio is not extremely small (e.g., $\gamma>1$ ), the attractive fixed point $(\text { ii) })_{c}$ [39] has a small value for electron-phonon interactions. Neither electron-electron nor electron-phonon interactions could induce the chargedensity wave. This conclusion explicitly conflicts with the Peierls phase transition [32, 62]. Because of an extremely small $\gamma$, the attractive fixed point $(\mathrm{ii})_{c}$ behaves like an infinitely large electron-phonon coupling point. Before reaching the attractive point $(\mathrm{ii})_{c}$, the electronphonon coupling becomes strong enough to induce the Peierls phase transition, which opens a charge-densitywave gap. Therefore, an extremely small $\gamma$ is crucial to induce the Peierls phase transition. In this case, electronphonon interactions can be viewed as a relevant perturbation starting from the Gaussian fixed point $(i)_{c}$ [see Fig. 3(b)]. The linearized renormalization-group equations around the fixed point $(\mathrm{i})_{c}$ show that the electronphonon coupling increases with $\ell$ as $e^{2 \ell}$. Therefore, the correlation length diverges with an exponent $\nu_{\beta}=1 / 2$ and the dynamical critical exponent $z=1[39,63]$. The general scaling relation of the resistivity to temperature and magnetic field is given by $[1,2,64]$

$$
\rho(B, T)=\rho\left(B_{c}\right) f\left[\left|B-B_{c}\right| / T^{1 / z \nu_{B}}\right],
$$
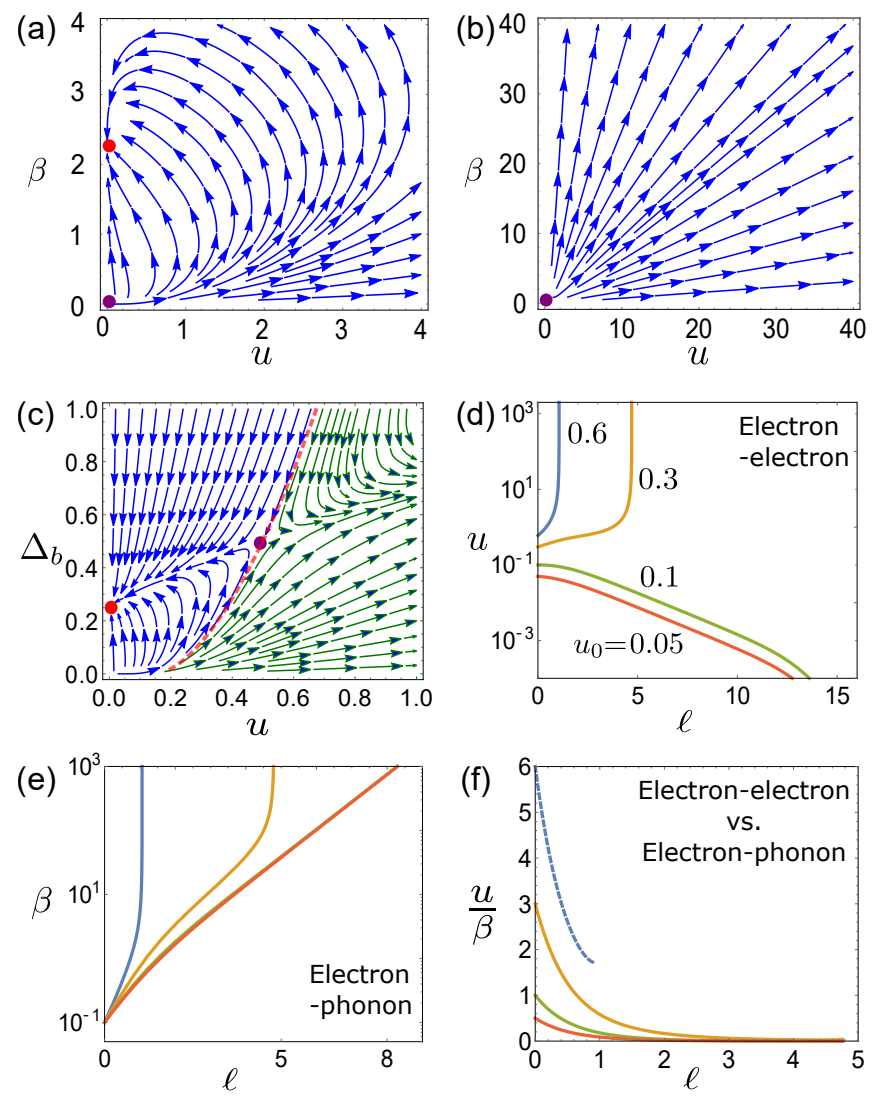

FIG. 3. The renormalization-group flow in the $u-\beta$ plane for (a) $\gamma=2$ and (b) $\gamma=0.002$. $u$ and $\beta$ measure the effective electron-electron and electron-phonon interactions, respectively. $\gamma=\left|v_{b} / v_{F}\right|$ is the ratio of the phonon speed to Fermi velocity. There are two fixed points at $(\mathrm{i})_{c}\left(u_{*}, \beta_{*}\right)=(0,0)$ and (ii) ${ }_{c}\left[0,(1+1 / \gamma)^{2}\right]$. (c) The renormalization-group flow in the $u-\Delta_{b}$ plane, in the absence of electron-phonon coupling. In this case, there are two non-Gaussian fixed points at $\left(u_{*}, \Delta_{b}^{*}\right)=(0,1 / 4)$ and $(1 / 2,1 / 2)$. The red curve distinguishes a disordered metal with zero electron-electron coupling on the left and a Wigner crystal on the right [39]. (d)-(f) Running of the electron-electron coupling $u$, electron-phonon coupling $\beta$, and $u / \beta$ with $\ell$, for different initial values of $u$. The initial values of both $\Delta_{b}$ and $\beta$ are 0.1 . (d)-(f) share the same legends. The green and red lines overlap in (e).

where $f(x)$ is a scaling function with $f(0)=1$. To obtain the general scaling with respect to the magnetic field, we have defined $\nu_{B}$, which describes how the correlation length $\zeta$ diverges with the magnetic field, following $\zeta \sim$ $\left|B-B_{c}\right|^{-\nu_{B}}$. On the other hand, the correlation length exponent obtained by our renormalization-group analysis describes that the correlation length $\zeta$ diverges with the coupling strength as $\zeta \sim\left|\beta-\beta_{c}\right|^{-\nu_{\beta}}$. Our scaling analysis based on Eq. (4) yields $\nu_{B}=3 \nu_{\beta}$ [39], and the critical exponent takes the form

$$
\xi=3 z \nu_{\beta}=1.5 .
$$

This value is far less than the experimental value $\xi=$ 5.5 [21], so without disorder, the coexistence of electron- 
electron and electron-phonon interactions cannot induce the metal-insulator transitions in the experiments [21].

Anderson localization induced by forward-scattering disorder?-When forward-scattering disorder exists, Anderson localization happens first with $z=1, \nu_{B}=2 \nu_{\Delta}=$ 2 , and $\xi=2$ (see details in Sec. SIIIE in [39]), which is far less than the experimental value $\xi=5.5$ [21].

Charge-density wave with backscattering disorder.When backscattering disorder exists, the fixed points are (i) ${ }_{b}\left(u_{*}, \beta_{*}, \Delta_{b}^{*}\right)=(0,0,0)$; (ii) $b\left[0,(1+1 / \gamma)^{2}, 0\right]$; (iii) $)_{b}$ $(0,0,1 / 4)$; and (iv) $)_{b}(1 / 2,0,1 / 2)$, belonging to four different universality classes because they have different critical exponents. Fixed point $(i)_{b}$ is unstable for electronphonon interactions and disorder. Fixed point (ii) $b$ is attractive for all three couplings and represents the chargedensity wave with an irrelevant electron-electron coupling and backscattering disorder [39]. Without electronphonon interactions, fixed point (iii) $)_{b}$ is stable and fixed point (iv) $)_{b}$ is a critical point for a Wigner crystal [39]. As shown in Fig. 3(c), there exists a critical line in the $u-\Delta_{b}$ plane; on the right, $u$ flows to infinity, indicating a charge-density wave driven by electron-electron interactions, i.e., a Wigner crystal, while on the left, the system flows to a finite disorder fixed point with a stable zero-valued electron-electron coupling. When including electron-phonon interactions, as shown in Fig. 3(d), the behavior of $u$ does not change qualitatively. Figure 3(e) shows that the electron-phonon coupling $\beta$ flows to infinity with increasing $\ell$. Near fixed point (iii) $)_{b}, \beta$ is the only unstable coupling. Fixed point $(\text { iii })_{b}$ becomes the critical fixed point for a charge-density wave induced by electron-phonon interactions in the presence of a finite backscattering disorder and irrelevant electron-electron coupling. The divergent $\beta$ gives $\nu_{\beta}=1$ [39]. The dynamical critical exponent for this universality class is found as $z=1+2 \Delta_{b}^{*}=1.5$. We thus get a critical exponent for the resistivity scaling

$$
\xi=3 z \nu_{\beta}=4.5
$$

which is close to the experimental value $\xi=5.5[21]$.

Near fixed point (iv) $)_{b}$, both $\beta$ and $u$ are unstable and increase unboundedly when the initial value of $u$ is large enough. Fixed point (iv) $b$ is a multicritical point for the Wigner crystal or Peierls phase transition. Which phase transition occurs first depends on the relative strength of $\beta$ and $u$. The solid lines in Fig. 3(e) show that the ratio of $u$ to $\beta$ decreases rapidly and finally vanishes when its initial value is not large enough, indicating that the Peierls phase transition happens first. The dotted line in Fig. 3(f) shows that $u$ is always larger than $\beta$, when its initial value is large enough, which means that the Wigner crystal occurs first. Despite that these two phase transitions both produce charge-density waves, the gap sizes and critical behaviors are different [31, 32]. For the Peierls phase transition, the correlation length exponent is $\nu_{\beta}=1$, and the dynamical critical exponent is $z=$
$1+2 \Delta_{b}^{*}=2$, which gives a critical exponent for the resistivity scaling:

$$
\xi=3 z \nu_{\beta}=6
$$

For the Wigner crystal, we obtain $\nu_{u}=1$ and $z=2$, which lead to $\xi=2$ [39].

Conclusion and experimental verification.-So far, $\xi=$ 6 is closest to the experimental result $\xi=5.5$ [21]. Therefore, we conclude that the magnetic-field-induced metalinsulator transition in the experiments is likely a chargedensity wave induced by electron-phonon interactions, in the presence of strong electron-electron interactions and backscattering disorder (only for samples 2-4 in Ref. [21]; see discussion below). Its quantum critical behavior is described by the universal class of fixed point (iv) $b$ with a correlation length exponent $\nu_{B}=3$ and dynamical critical exponent $z=2$.

Our conclusion can be verified by performing the current-scaling measurement [65-69], which along with the temperature scaling results in Ref. [21] could give rise to the experimental values of $z$ and $\nu$. The experimental setup is shown in Fig. 4(a). One needs to measure the longitudinal resistivity as a function of the magnetic field at different measurement currents $I$. Rearranging the measured magnetoresistivity $\rho(B) / \rho\left(B_{c}\right)$ near the critical field $B_{c}$ as a function of $\left|B-B_{c}\right| I^{-1 / \kappa}$ could generate a current-scaling plot as shown in Fig. 4(b), which is similar to that in Fig. 1 (b). The scaling relation of the resistivity to the current takes the form [2]

$$
\rho(B, T)=\rho\left(B_{c}\right) \mathcal{F}\left[\left|B-B_{c}\right| / I^{1 /(z+1) \nu_{B}}\right],
$$

where $\mathcal{F}(x)$ is a scaling function with $\mathcal{F}(0)=1$. With the fitted $\xi$ in Ref. [21] and $\kappa$ in this current-scaling measurement, the correlation length exponent $\nu_{B}$ and dynamical critical exponent $z$ can be found as

$$
\nu_{B}=\kappa-\xi, \quad z=\xi /(\kappa-\xi),
$$

respectively. Previously, this method has been widely employed in 2D quantum phase transitions [66-69], and it could provide an experimental way of distinguishing our theoretical exponents in Table I.

Also, the charge-density wave could be probed in X-ray diffraction spectrum [70-72], though the combination of the high magnetic field and X-ray facilities is challenging.

Discussion.-Our theory provides a deeper understanding to the data in Ref. [21], where the value $\xi=5.5$ is obtained by averaging four samples, as shown in Table I. However, the value of $\xi$ for sample 1 is closer to the value $\xi=4.5$ of the universal class described by fixed point (iii) ${ }_{b}$. By contrast, $\xi$ of samples $2-4$ belongs to fixed point (iv) ${ }_{b}$. The physical difference is that electron-electron interactions in sample 1 can be seen as zero valued when the metal-insulator transition happens, whereas they are finite and strong in the other three samples. As shown in Table I, the mean value of samples $2-4$ gives rise to 
(a)
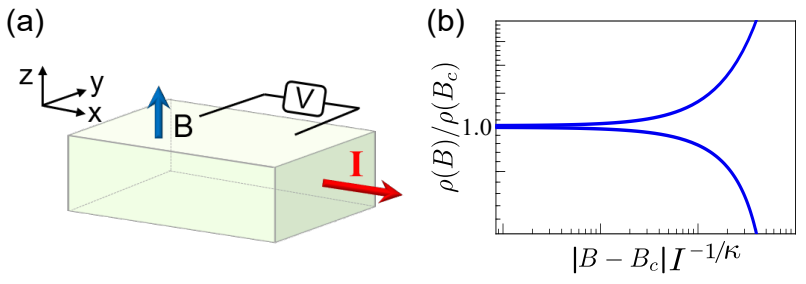

FIG. 4. (a) The experimental setup for the current-scaling measurement. The longitudinal resistivity $\rho \equiv V / I$ along the $x$ direction as a function of the magnetic field $B$ is measured at different measurement currents $I$ to construct a scaling function $\rho(B) / \rho\left(B_{c}\right)=\mathcal{F}\left(\left|B-B_{c}\right| / I^{1 / \kappa}\right)$ in (b), where $\mathcal{F}(x)$ is a scaling function with $\mathcal{F}(0)=1$. The linear fitting of the $\log \left(d \rho /\left.d B\right|_{B=B_{c}}\right)$ vs. $--\log (I)$ yields $1 / \kappa$. With the fitted $\xi$ in Fig. 1 and $\kappa$ here, the correlation length exponent $\nu_{B}=\kappa-\xi$ and the dynamical critical exponent $z=\xi /(\kappa-$ $\xi)$ can be found, to provide more experimental connections for our theory to identify the insulating ground state in the experiments.

$\xi=6.0$, which remarkably agrees with our theoretical value in Eq. (8). Despite that we take the experiments in Ref. [21] as a concrete sample to compare with, our theory can be applied to other 3D metal-insulator transitions under strong magnetic fields. Recently, the field-driven metal-insulator transition in $\beta-\mathrm{Bi}_{4} \mathrm{I}_{4}$ [73] is found to have $\xi=6.5$ within the experimental error, showing the wide application potential of our theory. Nevertheless, 3D metal-insulator transitions of spin-correlated systems driven by magnetic fields [18-20] are beyond the scope of our theory and will be a challenging topic in the future.

We thank helpful discussions with Liyuan Zhang and Tingyu Gao. This work was supported by the National Natural Science Foundation of China (11925402 and 12047531), the National Basic Research Program of China (2015CB921102), the Strategic Priority Research Program of Chinese Academy of Sciences (XDB28000000), Guangdong province (2020KCXTD001 and 2016ZT06D348), Shenzhen High-level Special Fund (G02206304 and G02206404), and the Science, Technology and Innovation Commission of Shenzhen Municipality (ZDSYS20170303165926217, JCYJ20170412152620376, and KYTDPT20181011104202253). The numerical calculations were supported by Center for Computational Science and Engineering of SUSTech.

* Corresponding author: luhz@sustech.edu.cn

[1] M. Imada, A. Fujimori, and Y. Tokura, Metal-insulator transitions, Rev. Mod. Phys. 70, 1039 (1998).

[2] S. L. Sondhi, S. M. Girvin, J. P. Carini, and D. Shahar, Continuous quantum phase transitions, Rev. Mod. Phys. 69, 315 (1997).

[3] S. V. Kravchenko and M. P. Sarachik, Metal-insulator transition in two-dimensional electron systems, Rep. Prog. Phys. 67, 1 (2003).

[4] D. J. Newson and M. Pepper, Critical conductivity at the magnetic-field-induced metal-insulator transition in n-GaAs and n-InSb, J. Phys. C 19, 3983 (1986).

[5] V. J. Goldman, M. Shayegan, and H. D. Drew, Anomalous Hall Effect below the Magnetic-Field-Induced MetalInsulator Transition in Narrow-Gap Semiconductors, Phys. Rev. Lett. 57, 1056 (1986).

[6] M. C. Maliepaard, M. Pepper, R. Newbury, J. E. F. Frost, D. C. Peacock, D. A. Ritchie, G. A. C. Jones, and G. Hill, Evidence of a magnetic-field-induced insulatormetal-insulator transition, Phys. Rev. B 39, 1430 (1989).

[7] P. Dai, Y. Zhang, and M. P. Sarachik, Effect of a Magnetic Field on the Critical Conductivity Exponent at the Metal-Insulator Transition, Phys. Rev. Lett. 67, 136 (1991).

[8] S. Kivelson, D.-H. Lee, and S.-C. Zhang, Global phase diagram in the quantum Hall effect, Phys. Rev. B 46, 2223 (1992).

[9] T. Wang, K. P. Clark, G. F. Spencer, A. M. Mack, and W. P. Kirk, Magnetic-Field-Induced Metal-Insulator Transition in Two Dimensions, Phys. Rev. Lett. 72, 709 (1994).

[10] Y. Tomioka, A. Asamitsu, H. Kuwahara, Y. Moritomo, and Y. Tokura, Magnetic-field-induced metal-insulator phenomena in $\mathrm{Pr}_{1-x} \mathrm{Ca}_{x} \mathrm{MnO}_{3}$ with controlled chargeordering instability, Phys. Rev. B 53, R1689 (1996).

[11] D. Popović, A. B. Fowler, and S. Washburn, MetalInsulator Transition in Two Dimensions: Effects of Disorder and Magnetic Field, Phys. Rev. Lett. 79, 1543 (1997).

[12] X. C. Xie, X. R. Wang, and D. Z. Liu, KosterlitzThouless-Type Metal-Insulator Transition of a 2D Electron Gas in a Random Magnetic Field, Phys. Rev. Lett. 80, 3563 (1998).

[13] J. An, C. D. Gong, and H. Q. Lin, Theory of the magnetic-field-induced metal-insulator transition, Phys. Rev. B 63, 174434 (2001).

[14] H. Kempa, P. Esquinazi, and Y. Kopelevich, Fieldinduced metal-insulator transition in the c-axis resistivity of graphite, Phys. Rev. B 65, 241101(R) (2002).

[15] E. V. Gorbar, V. P. Gusynin, V. A. Miransky, and I. A. Shovkovy, Magnetic field driven metal-insulator phase transition in planar systems, Phys. Rev. B 66, 045108 (2002).

[16] Y. Kopelevich, J. C. Medina Pantoja, R. R. da Silva, and S. Moehlecke, Universal magnetic-field-driven metalinsulator-metal transformations in graphite and bismuth, Phys. Rev. B 73, 165128 (2006).

[17] D. J. W. Geldart and D. Neilson, Quantum critical behavior in the insulating region of the two-dimensional metal-insulator transition, Phys. Rev. B 76, 193304 (2007).

[18] S. Calder, V. O. Garlea, D. F. McMorrow, M. D. Lumsden, M. B. Stone, J. C. Lang, J.-W. Kim, J. A. Schlueter, Y. G. Shi, K. Yamaura, Y. S. Sun, Y. Tsujimoto, and A. D. Christianson, Magnetically Driven Metal-Insulator Transition in $\mathrm{NaOsO}_{3}$, Phys. Rev. Lett. 108, 257209 (2012).

[19] K. Ueda, J. Fujioka, B.-J. Yang, J. Shiogai, A. Tsukazaki, S. Nakamura, S. Awaji, N. Nagaosa, and Y. Tokura, Magnetic Field-Induced Insulator-Semimetal Transition in a Pyrochlore $\mathrm{Nd}_{2} \mathrm{Ir}_{2} \mathrm{O}_{7}$, Phys. Rev. Lett. 115, 056402 (2015). 
[20] Z. Tian, Y. Kohama, T. Tomita, H. Ishizuka, T. H. Hsieh, J. J. Ishikawa, K. Kindo, L. Balents, and S. Nakatsuji, Field-induced quantum metal-insulator transition in the pyrochlore iridate $\mathrm{Nd}_{2} \mathrm{Ir}_{2} \mathrm{O}_{7}$, Nat. Phys. 12, 134 (2016).

[21] F. Tang, Y. Ren, P. Wang, R. Zhong, J. Schneeloch, S. A. Yang, K. Yang, P. A. Lee, G. Gu, Z. Qiao, and L. Zhang, Three-dimensional quantum Hall effect and metal-insulator transition in $\mathrm{ZrTe}_{5}$, Nature (London) 569, 537 (2019).

[22] P. Wang, Y. Ren, F. Tang, P. Wang, T. Hou, H. Zeng, L. Zhang, and Z. Qiao, Approaching three-dimensional quantum Hall effect in bulk HfTe ${ }_{5}$, Phys. Rev. B 101, 161201(R) (2020).

[23] M. Vojta, Quantum phase transitions, Rep. Prog. Phys. 66, 2069 (2003).

[24] S. Sachdev, Quantum Phase Transitions, 2nd ed. (Cambridge University Press, Cambridge, England, 2011).

[25] H. v. Löhneysen, A. Rosch, M. Vojta, and P. Wölfle, Fermi-liquid instabilities at magnetic quantum phase transitions, Rev. Mod. Phys. 79, 1015 (2007).

[26] E. Wigner, On the Interaction of Electrons in Metals, Phys. Rev. 46, 1002 (1934).

[27] S. B. Field, D. H. Reich, B. S. Shivaram, T. F. Rosenbaum, D. A. Nelson, and P. B. Littlewood, Evidence for depinning of a Wigner crystal in $\mathrm{Hg}-\mathrm{Cd}-\mathrm{Te}$, Phys. Rev. B 33, 5082 (1986).

[28] P. W. Anderson, Absence of Diffusion in Certain Random Lattices, Phys. Rev. 109, 1492 (1958).

[29] E. Abrahams, P. W. Anderson, D. C. Licciardello, and T. V. Ramakrishnan, Scaling Theory of Localization: Absence of Quantum Diffusion in Two Dimensions, Phys. Rev. Lett. 42, 673 (1979).

[30] F. Evers and A. D. Mirlin, Anderson transitions, Rev. Mod. Phys. 80, 1355 (2008).

[31] T. Giamarchi, Quantum Physics in One Dimension (Clarendon Press, Oxford, 2003).

[32] G. Grüner, Density Waves in Solids (Westview Press, Boulder, CO, 2000).

[33] F. J. Wegner, Electrons in disordered systems. Scaling near the mobility edge, Z. Phys. B 25, 327 (1976).

[34] W. L. McMillan, Scaling theory of the metal-insulator transition in amorphous materials, Phys. Rev. B 24, 2739 (1981).

[35] C. A. Stafford and A. J. Millis, Scaling theory of the Mott-Hubbard metal-insulator transition in one dimension, Phys. Rev. B 48, 1409 (1993).

[36] B. Huckestein, Scaling theory of the integer quantum Hall effect, Rev. Mod. Phys. 67, 357 (1995).

[37] V. Dobrosavljević, E. Abrahams, E. Miranda, and S. Chakravarty, Scaling Theory of Two-Dimensional Metal-Insulator Transitions, Phys. Rev. Lett. 79, 455 (1997).

[38] A. Pelissetto and E. Vicari, Critical phenomena and renormalization-group theory, Phys. Rep. 368, 549 (2002).

[39] See Supplemental Material at ... for detailed calculations, which includes Refs.[1,2,21,31,32,40,42-44,54-64].

[40] F. Qin, S. Li, Z. Z. Du, C. M. Wang, W. Zhang, D. Yu, H.-Z. Lu, and X. C. Xie, Theory for the Charge-DensityWave Mechanism of 3D Quantum Hall Effect, Phys. Rev. Lett. 125, 206601 (2020).

[41] S.-Q. Shen, Topological Insulators, 2nd ed. (SpringerVerlag, Berlin, 2017).

[42] R. Y. Chen, Z. G. Chen, X.-Y. Song, J. A. Schneeloch,
G. D. Gu, F. Wang, and N. L. Wang, Magnetoinfrared Spectroscopy of Landau Levels and Zeeman Splitting of Three-Dimensional Massless Dirac Fermions in $\mathrm{ZrTe}_{5}$, Phys. Rev. Lett. 115, 176404 (2015).

[43] Y. Jiang, Z. L. Dun, H. D. Zhou, Z. Lu, K.-W. Chen, S. Moon, T. Besara, T. M. Siegrist, R. E. Baumbach, D. Smirnov, and Z. Jiang, Landau-level spectroscopy of massive Dirac fermions in single-crystalline $\mathrm{ZrTe}_{5}$ thin flakes, Phys. Rev. B 96, 041101(R) (2017).

[44] S.-K. Jian and Z. Zhu, $2 k_{F}$ density wave instability of composite Fermi liquid, Phys. Rev. Research 2, 033414 (2020).

[45] H. Bruus, K. Flensberg, and O. U. Press, Many-Body Quantum Theory in Condensed Matter Physics: An Introduction (Oxford University Press, Oxford, 2004).

[46] F. Wegner, The mobility edge problem: Continuous symmetry and a conjecture, Z. Phys. B 35, 207 (1979).

[47] K. B. Efetov, A. I. Larkin, and D. E. Khmel'nitskii, Interaction of diffusion modes in the theory of localization, Sov. Phys. JETP 52, 568 (1980).

[48] P. A. Lee and T. V. Ramakrishnan, Disordered electronic systems, Rev. Mod. Phys. 57, 287 (1985).

[49] T. Giamarchi and H. J. Schulz, Anderson localization and interactions in one-dimensional metals, Phys. Rev. B 37, 325 (1988).

[50] M. E. Fisher, The renormalization group in the theory of critical behavior, Rev. Mod. Phys. 46, 597 (1974).

[51] K. G. Wilson, The renormalization group and critical phenomena, Rev. Mod. Phys. 55, 583 (1983).

[52] A. MacKinnon, Critical exponents for the metal-insulator transition, J. Phys.: Condens. Matter 6, 2511 (1994).

[53] H. E. Stanley, Scaling, universality, and renormalization: Three pillars of modern critical phenomena, Rev. Mod. Phys. 71, S358 (1999).

[54] K. G. Wilson and J. Kogut, The renormalization group and the $\epsilon$ expansion, Phys. Rep. 12, 75 (1974).

[55] A. A. Abrikosov, L. P. Gorkov, and I. E. Dzyaloshinski, Methods of Quantum Field Theory in Statistical Physics (Dover, New York, 1975).

[56] M. E. Peskin and D. V. Schroeder, An Introduction to Quantum Field Theory (CRC Press, Boca Raton, FL, 2019).

[57] G. D. Mahan, Many-Particle Physics (Kluwer Academic/Plenum Publishers, New York, 2000).

[58] R. Shankar, Renormalization-group approach to interacting fermions, Rev. Mod. Phys. 66, 129 (1994).

[59] J. Zhu, T. Feng, S. Mills, P. Wang, X. Wu, L. Zhang, S. T. Pantelides, X. Du, and X. Wang, Record-Low and Anisotropic Thermal Conductivity of a Quasi-OneDimensional Bulk ZrTes Single Crystal, ACS Appl. Mater. Interfaces 10, 40740 (2018).

[60] A. A. Abrikosov, Quantum magnetoresistance, Phys. Rev. B 58, 2788 (1998).

[61] H.-Z. Lu, S.-B. Zhang, and S.-Q. Shen, High-field magnetoconductivity of topological semimetals with shortrange potential, Phys. Rev. B 92, 045203 (2015).

[62] W. Kohn, Image of the Fermi Surface in the Vibration Spectrum of a Metal, Phys. Rev. Lett. 2, 393 (1959).

[63] P. Goswami and S. Chakravarty, Quantum Criticality between Topological and Band Insulators in $3+1$ Dimensions, Phys. Rev. Lett. 107, 196803 (2011).

[64] M. P. A. Fisher, Quantum Phase Transitions in Disordered Two-Dimensional Superconductors, Phys. Rev. Lett. 65, 923 (1990). 
[65] D. G. Polyakov and B. I. Shklovskii, Conductivity-peak broadening in the quantum Hall regime, Phys. Rev. B 48, 11167 (1993).

[66] H. P. Wei, L. W. Engel, and D. C. Tsui, Current scaling in the integer quantum Hall effect, Phys. Rev. B 50, 14609 (1994).

[67] S. V. Kravchenko, D. Simonian, M. P. Sarachik, W. Mason, and J. E. Furneaux, Electric Field Scaling at a $B=0$ Metal-Insulator Transition in Two Dimensions, Phys. Rev. Lett. 77, 4938 (1996).

[68] W. Pan, D. Shahar, D. C. Tsui, H. P. Wei, and M. Razeghi, Quantum Hall liquid-to-insulator transition in $\mathrm{In}_{1-\mathrm{x}} \mathrm{Ga}_{\mathrm{x}} \mathrm{As} / \mathrm{InPt}$ heterostructures, Phys. Rev. B 55, 15431 (1997).

[69] M. Salehi, H. Shapourian, I. T. Rosen, M.-G. Han, J. Moon, P. Shibayev, D. Jain, D. Goldhaber-Gordon, and S. Oh, Quantum-Hall to Insulator Transition in Ultra-Low-Carrier-Density Topological Insulator Films and a Hidden Phase of the Zeroth Landau Level, Adv.
Mater. 31, 1901091 (2019).

[70] S. Wang, A. E. Kovalev, A. V. Suslov, and T. Siegrist, A facility for X-ray diffraction in magnetic fields up to $25 \mathrm{~T}$ and temperatures between 15 and $295 \mathrm{~K}$, Rev. Sci. Instrum. 86, 123902 (2015).

[71] Y. Narumi, K. Kindo, K. Katsumata, M. Kawauchi, C. Broennimann, U. Staub, H. Toyokawa, Y. Tanaka, K. Kikkawa, T. Yamamoto, M. Hagiwara, T. Ishikawa, and H. Kitamura, X-ray diffraction studies in pulsed high magnetic fields, J. Phys.: Conf. Ser. 51, 494 (2006).

[72] P. Pototsching, E. Gratz, H. Kirchmayr, and A. Lindbaum, X-ray diffraction in magnetic fields, J. Alloys Compd. 247, 234 (1997).

[73] P. Wang, F. Tang, P. Wang, H. Zhu, C.-W. Cho, J. Wang, X. Du, Y. Shao, and L. Zhang, Quantum transport properties of $\beta-\mathrm{Bi}_{4} \mathrm{I}_{4}$ near and well beyond the extreme quantum limit, Phys. Rev. B 103, 155201 (2021). 